\title{
Neuronal Circuits Modulate Antigen Flow Through Lymph Nodes
}

\author{
William M Hanes, ${ }^{1,2}$ Peder S Olofsson, ${ }^{3}$ Sébastien Talbot, ${ }^{4,5}$ Tea Tsaava, ${ }^{1}$ Mahendar Ochani, ${ }^{1,6}$ \\ Gavin H Imperato, ${ }^{1}$ Yaakov A Levine, ${ }_{,}^{1,7}$ Jesse Roth, ${ }^{1}$ Maud A Pascal,,${ }^{4,5}$, Simmie L Foster, ${ }^{4,5}$ Ping Wang, ${ }^{6}$ \\ Clifford Woolf, ${ }^{4,5}$ Sangeeta S Chavan, ${ }^{1,3^{*}}$ and Kevin J Tracey ${ }^{1,3^{*}}$
}

${ }^{1}$ Laboratory of Biomedical Science, The Feinstein Institute for Medical Research, Manhasset, New York, United States of America; ${ }^{2}$ Department of Biochemistry and Cell Biology, Stony Brook University, Stony Brook, New York, United States of America; ${ }^{3}$ Center for Bioelectronic Medicine, Department of Medicine, Center for Molecular Medicine, Solna, Karolinska Institutet, Karolinska University Hospital, Stockholm, Sweden; ${ }^{4}$ FM Kirby Neurobiology Center, Children's Hospital Boston, Boston, Massachusetts, United States of America; ${ }^{5}$ Department of Neurobiology, Harvard Medical School, Boston, Massachusetts, United States of America; ${ }^{6}$ Division of Surgical Research, The Feinstein Institute for Medical Research, Manhasset, New York, United States of America; ${ }^{7}$ SetPoint Medical Corporation, Valencia, California, United States of America; and ${ }^{8}$ Département de biologie, École Normale Supérieure de Cachan, Cachan, France

\begin{abstract}
When pathogens and toxins breech the epithelial barrier, antigens are transported by the lymphatic system to lymph nodes. In previously immunized animals, antigens become trapped in the draining lymph nodes, but the underlying mechanism that controls antigen restriction is poorly understood. Here we describe the role of neurons in sensing and restricting antigen flow in lymph nodes. The antigen keyhole-limpet hemocyanin $(K L H)$ injected into the mouse hind paw flows from the popliteal lymph node to the sciatic lymph node, continuing through the upper lymphatics to reach the systemic circulation. Re-exposure to KLH in previously immunized mice leads to decreased flow from the popliteal to the sciatic lymph node as compared with nailve mice. Administering bupivacaine into the lymph node region restores antigen flow in immunized animals. In contrast, neural activation using magnetic stimulation significantly decreases antigen trafficking in naïve animals as compared with sham controls. Ablating $\mathrm{Na}_{\mathrm{V}} 1.8+$ sensory neurons significantly reduces antigen restriction in immunized mice. Genetic deletion of Fc $\gamma \mathrm{RI} / \mathrm{Fc} \varepsilon \mathrm{RI}$ also reverses the antigen restriction. Colocalization of PGP9.5-expressing neurons, FcyRl receptors and labeled antigen occurs at the antigen challenge site. Together, these studies reveal that neuronal circuits modulate antigen trafficking through a pathway that requires $\mathrm{Na}_{\mathrm{V}} 1.8$ and Fc $\gamma \mathrm{R}$.
\end{abstract}

Online address: www.bioelecmed.org

doi: 10.15424/bioelectronmed.2016.00001

\section{INTRODUCTION}

The immune and nervous systems coevolved under the threat of environmental dangers and work in concert to sense changes in the environment, mobilize host responses and establish memories of threatening events. The nervous system communicates with the immune system via direct innervation of primary and secondary lymphoid organs. These lymphoid organs play an essential role in peripheral inflammatory responses to immunological challenge and/or tissue injury. Antigens are transported to the local lymph nodes, processed by antigen-presenting cells and presented

${ }^{*}$ SSC and KJT contributed equally as senior authors.

Address correspondence to Sangeeta S Chavan, The Feinstein Institute for Medical Research, Northwell Health, 350 Community Drive, Manhasset, NY 11030 USA;

Phone: 516-562-2118; Fax: 516-562-1022; E-mail: schavan@northwell.edu

Submitted January 21, 2016; Accepted for Publication December 5, 2016;

Published Online (wWw.molmed.org) December 20, 2016.

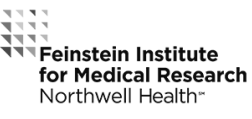

for Medical Research

Northwell Health 
targeting single antigen-presenting cells (2). Cross-talk between local neural and immune responses modulates inflammatory and neural input to lymph nodes. Antigenic stimulation leads to an increase in the density of lymph node innervation (3). In a contact sensitivity model, antigenic challenge induces an increase in the length of nerve fibers (4). Subcutaneous injection of antigen is associated with increased nerve-ending density in the medulla of the draining lymph node, indicating functional activation of the autonomic nervous system through the immune system (5). Catecholamine released during acute psychological stress leads to mobilization of lymphocytes into the blood (6). Direct neural stimulation leads to increased output of lymphocytes from the popliteal lymph nodes (7). In contrast, silencing local neural input reduces lymphocyte output from lymph nodes by more than $99 \%$, indicating that neural signals control leukocyte retention and trafficking through the lymph nodes (8).

Recent studies have demonstrated that sensory nociceptor neurons that express the ion channels TRPV1 and $\mathrm{Na}_{\mathrm{V}} 1.8$ are activated directly by bacteria, leading to modulation of pain and inflammation (9). These nociceptors are also essential for developing allergic inflammatory responses in allergic conditions and cutaneous inflammation (10-12). Nociceptors have been implicated in modulating lymph node function, because ablation of nociceptor neurons attenuates hypertrophy in draining lymph nodes and skin inflammatory responses in psoriasis (12). Specific ablation of $\mathrm{Na}_{\mathrm{V}}$ 1.8-lineage nociceptor neurons abrogates pain during bacterial infection, but concurrently increases local immune infiltration and lymphadenopathy in the draining lymph nodes (9). Together these observations indicate that nociceptor neurons are activated by exogenous stimuli and modulate lymph node trafficking. However, the specific subset of peripheral neurons that control the lymphatic transit has not yet been characterized. Here, we investigated the transit of a labeled antigen through the lymphatic system and identified the TRPV1 ${ }^{-} / \mathrm{Na}_{\mathrm{V}} 1.8^{+}$population of peripheral neurons as required for neuronal input leading to antigen restriction.

\section{MATERIALS AND METHODS}

\section{Antigen Labeling}

Keyhole limpet hemocyanin (KLH, Calbiochem) and ovalbumin (OVA, Sigma-Aldrich) were diluted to $1 \mathrm{mg} / \mathrm{mL}$ with phosphate-buffered saline (PBS), then potassium phosphate dibasic, $\mathrm{pH} 9.0$, was added to $0.1 \mathrm{M}$ final concentration. Then $0.5 \mathrm{mg}$ IrDye (800CW-NHS ester or 680LT-NHS ester, Licor) or Alexa Fluor ${ }^{\circledR} 647$ NHS Ester (A647, Thermo Scientific) was added for each $25 \mathrm{mg}$ protein; $1 \mathrm{~mL}$ aliquots were then incubated at $20^{\circ} \mathrm{C}$ with $300 \mathrm{RPM}$, shaking for $2 \mathrm{~h}$. Labelled antigen was separated by centrifugation through PBS-washed Zeba spin desalting columns (Thermo Scientific). Dye-labeled antigens were concentrated with $100 \mathrm{~K}(\mathrm{KLH})$ or $30 \mathrm{~K} \mathrm{MWCO}$ (OVA) spin columns (Millipore).

\section{Gel Electrophoresis}

Labeled antigens were loaded on NuPAGE 4-12\% Bis-Tris Gels (Invitrogen) with NuPAGE MOPS SDS Running Buffer and run according to the manufacturer's instructions, $50 \mathrm{~min}$ at 200 constant volts. Gels were extracted from cassettes and imaged directly on an Odyssey infrared imager (Licor).

\section{Animals}

Balb/c, B6.129P2-Gt(ROSA)26Sor ${ }^{\text {tm1(DTA)Lky }}$ (floxed-stop-DTA), B6.129-Trpv1 ${ }^{\mathrm{tm} 1 \text { (cre)Bbm }}$ (TRPV1-cre) and B6;129P2-Fcer1g ${ }^{\text {tm1Rav }}$ (FcR KO) mice were obtained from Jackson Laboratories. FcR KO mice lack the Fcy adaptor protein, which transduces Fc $\gamma$ RI and FceRI signaling. $\mathrm{Na}_{\mathrm{V}} 1.8-\mathrm{Cre}^{+/-}$mice (13) were a gift to $\mathrm{CW}$ from R. Kuner (University of Heidelberg). $\mathrm{Na}_{\mathrm{V}} 1.8-\mathrm{Cre}^{+/-}$mice were bred with C57BL/6 $\mathrm{DTA}^{+/+}$mice to generate nociceptor-deficient $\mathrm{Na}_{\mathrm{V}} 1$.8$\mathrm{Cre}^{+/-} / \mathrm{DTA}^{+/-}$and control littermates $\left(\mathrm{Na}_{\mathrm{V}} 1.8-\mathrm{Cre}^{-/-} / \mathrm{DTA}^{+/-}\right)$. TRPV1-Cre ${ }^{+/-}$ mice were bred with C57BL/6 $\mathrm{DTA}^{+/+}$ mice to generate TRPV1-Cre ${ }^{+/-} / \mathrm{DTA}^{+/-}$ and control littermates (TRPV1-Cre ${ }^{-/-} /$ $\left.\mathrm{DTA}^{+/-}\right)$. Food and water were available ad libitum. Mice were used in subsequent experiments after at least a $14 \mathrm{~d}$ adaptation period. All procedures were performed in accordance with National Institutes of Health Guidelines (14) under protocols approved by the Institutional Animal Care and Use Committee of The Feinstein Institute for Medical Research.

\section{Tissue Staining}

Mice were immunized with $100 \mu \mathrm{g}$ antigen and 50\% Imject alum in $200 \mu \mathrm{l}$ saline, injected intraperitoneally twice, 2 wks apart. Two weeks after the second injection, mice were injected with KLHA647 in the dorsum of the hind paw. After $1 \mathrm{~h}$, skin around the injection site was excised, frozen in optimal cutting temperature media (Tissue-Tek), sliced at $10 \mu \mathrm{m}$ and mounted on Superfrost/ Plus slides (Fisher Scientific). Sections were blocked with 5\% goat serum and rat anti-CD16/CD32 (BD Biosciences). Sections were then stained for $1 \mathrm{~h}$ with rabbit anti-PGP9.5 (EMD Millipore) and mouse anti-CD64 (Biolegend, clone $\times 54-5 / 7.1$ ). Secondary antibodies were goat anti-mouse IgG-Dylight 550 and goat anti-rabbit IgG-Dylight 488 (Thermo Scientific). Images were obtained on a FluoView FV300 laser-scanning confocal microscope (Olympus).

\section{Immunization}

Unlabeled antigen $(100 \mu \mathrm{g})$ and 50\% Imject alum (Thermo Scientific) in $200 \mu 1$ of $0.9 \%$ saline was injected intraperitoneally. Two weeks later, animals were injected again with the same antigen emulsion as a booster. Two weeks after the booster injection, animals were studied in experiments. Control animals received injections of $50 \%$ alum in saline. For passive immunization, naïve Balb/c mice were injected intraperitoneally with $100 \mu \mathrm{g}$ rabbit polyclonal anti-ovalbumin antibody (Millipore). After $24 \mathrm{~h}$, mice were injected with OVA-800CW or KLH$800 \mathrm{CW}$ subcutaneously in the dorsum of the hind paw. 


\section{In Situ Antigen Imaging}

Using an insulin syringe, $20 \mu \mathrm{l}$ of $100 \mu \mathrm{g}$ labeled antigen was injected subcutaneously into the dorsum of the hind paw. At the end of the experiment, the animal was euthanized using carbon dioxide asphyxiation, the skin over the area of interest was removed and the animal was placed in the supine position in an Odyssey infrared imager to detect the fluorescence of labeled antigen in the popliteal and sciatic lymph nodes. Fluorescent antigen signal was expressed as arbitrary units (A.U.). To image lymph node antigen content, sciatic and popliteal lymph nodes were surgically removed and placed into black, clear-bottomed 96-well plates filled with T-per (Thermo Scientific). These plates were imaged on the Odyssey imager.

\section{Antigen-Specific Antibody Titer}

High-binding 96-well microplates (Corning) were coated using $20 \mu \mathrm{g} / \mathrm{mL}$ KLH in PBS and incubated overnight at room temperature. The following day, the plates were washed with PBS $+0.01 \%$ Tween 20 and blocked with $1 \mathrm{mg} / \mathrm{mL}$ bovine serum albumin in PBS. Serum from immunized animals was obtained by cardiac puncture followed by centrifugation at $2,000 \times g$ for $10 \mathrm{~min}$. Serum was diluted 1:100-1:10,000 with PBS and $100 \mu \mathrm{l}$ per sample was added to washed and blocked plates. Plates were incubated for $2 \mathrm{~h}$, followed by incubation with 1:2000 sheep-derived anti-mouse IgM-HRP (BD) or anti-mouse IgG (Amersham) for $2 \mathrm{~h}$. Plates were washed a final time, developed using Opt-EIA (BD) and the reaction was stopped with $\mathrm{H}_{2} \mathrm{SO}_{4}$.

\section{Nerve Block}

Mice were immunized with $100 \mu \mathrm{g}$ antigen and 50\% Imject alum in $200 \mu \mathrm{l}$ saline injected intraperitoneally twice, 2 wks apart. Two weeks after the second injection, $25 \mu \mathrm{l}$ bupivacaine $(0.375 \%$, APP Pharmaceuticals) or $0.9 \%$ saline was injected at the sciatic and femoral nerves of the hind leg. Twenty minutes after the initial injection, animals were injected with labeled antigen in the dorsum of the hind paw.

\section{Electrical Nerve Stimulation}

Mice were anesthetized with isoflurane in the prone position. A 28-gauge uncoated grounding electrode (Technomed) was placed subcutaneously at the top of the thigh, and a coated needle electrode (Alpine Biomed) was inserted by the popliteal lymph node. Stimulation was delivered by a Biopac stimulation module controlled with AcqKnowledge 4.1 software. Parameters were $-5 \mathrm{~V}$ constant, $0.75 \mathrm{msec}$ pulse duration, $20 \mathrm{~Hz}$ (50 msec period). Stimulation was applied for $5 \mathrm{~min}$; after the first minute, KLH-800CW was injected into the dorsum of the hind paw.

\section{Magnetic Nerve Stimulation}

Mice were anesthetized with isoflurane in the prone position. Magnetic stimulation was administered using an MC-B35 butterfly coil driven by a MagPro stimulator (Magventure) aimed at the popliteal and sciatic lymph nodes. The parameters used were $50 \%$ power, 120 pulses $(2 \mathrm{~Hz}$, $60 \mathrm{~s}$ ) train 1, $1 \mathrm{~s}$ train interval. Control animals were anesthetized and the magnetic coil was positioned, but no current was applied. Immediately after stimulation, mice were removed from the table, then KLH-800CW was injected into the dorsum of the hind paw.

\section{Statistics}

Antigen concentration compared with fluorescence and in situ compared with in-plate fluorescence were analyzed by linear regression. Control and experimental popliteal and sciatic lymph node signals were individually analyzed by unpaired Student $t$ test. Antigen concentration curves and control/TRPV1/ $\mathrm{Na}_{\mathrm{V}} 1.8$ lymph node fluorescence were analyzed by one-way analysis of variance (ANOVA) followed by Bonferroni post-test. All tests with $p<.05$ were considered statistically significant. Statistical analyses were performed using Graphpad Prism 6 software. Unless otherwise stated, all numbers are given as average \pm standard error of the mean; $\mathrm{n}$ represents the number of mice used in each group. In graphs, ${ }^{*}$ indicates $p<.05$,
** indicates $p<.01, * * *$ indicates $p<.001$ and ${ }^{* * * *}$ indicates $p<.0001$.

\section{RESULTS}

\section{Antigen Flow Through the Lymphatic Chain Was Monitored with Infrared Fluorescent Dye-Labeled Antigen}

To monitor antigen localization and concentration in the peripheral lymphatic tissue, we utilized antigens linked to near-infrared fluorescent dyes. This allowed in situ imaging of the lymphatic system in whole animals using a Licor Odyssey flatbed imager with low background and relatively high resolution, giving a view of the involved lymphatic system as a whole. To validate this imaging method, we first imaged a range of concentrations of dye-labeled antigen on polyacrylamide gel. A linear correlation between antigen concentration and fluorescent signal was found upon imaging the gel on a Licor Odyssey infrared plate imager (Figures 1A, B). Next we determined whether a dose-dependent fluorescent signal could be detected from the foot of mouse. A range of concentrations of labeled antigen was injected in the dorsum of the hind paw of a mouse, and trafficking of the labeled antigen from the foot to the lymphatic system was imaged using the Licor Odyssey infrared plate imager. A dose-dependent increase in accumulation of the labeled antigen in the popliteal (Figure 1C) and sciatic (Figure 1D) lymph nodes was observed. Notably, a similar dosedependent fluorescent signal was observed in the popliteal (Figure 1E) and sciatic (Figure 1F) lymph nodes when lymph nodes were extracted and imaged in a 96-well plate. To minimize the variability observed in antigen signal (20-70, Figure $1 F)$, all the groups in an experiment were analyzed on the same day. A linear correlation with an R-squared value of 0.7634 was found when plotting the in situ fluorescent signal against the signal in the 96-well plate (Figure 1G). These data indicate that fluorescently labeled antigen can 


\section{A}

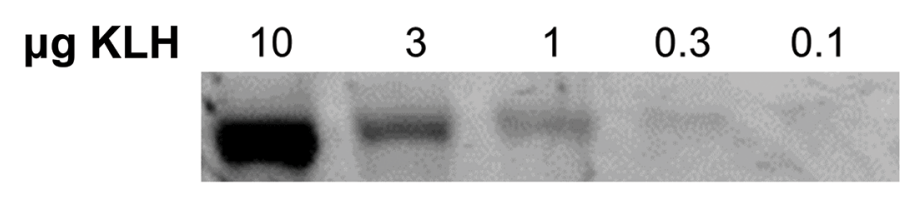

B

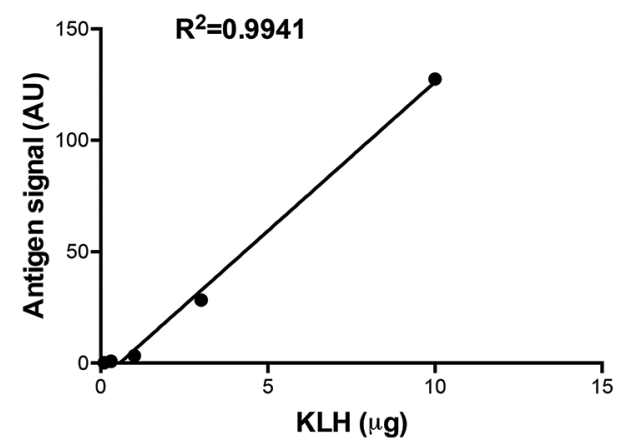

D

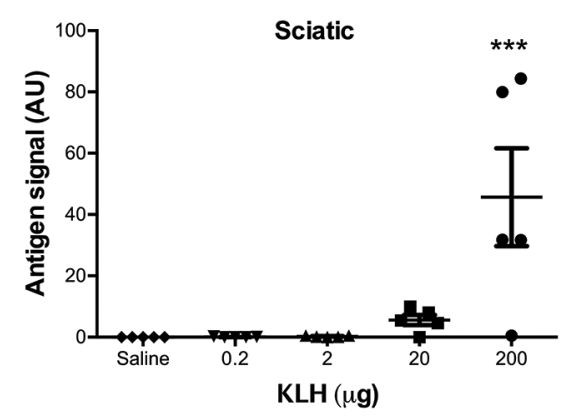

$\mathrm{F}$

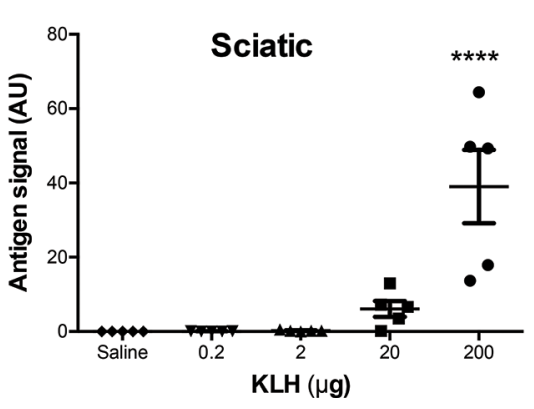

C

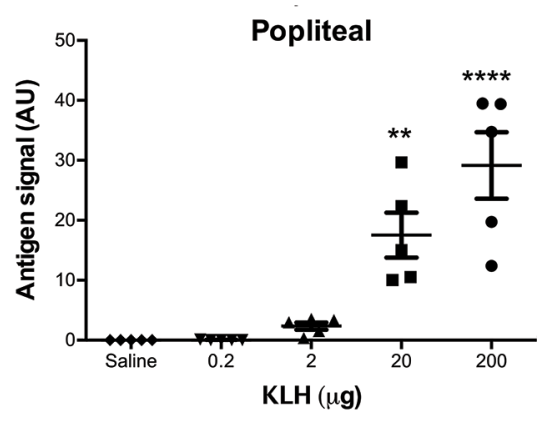

E

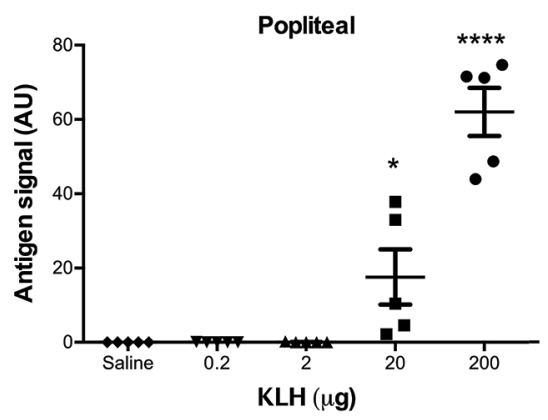

G

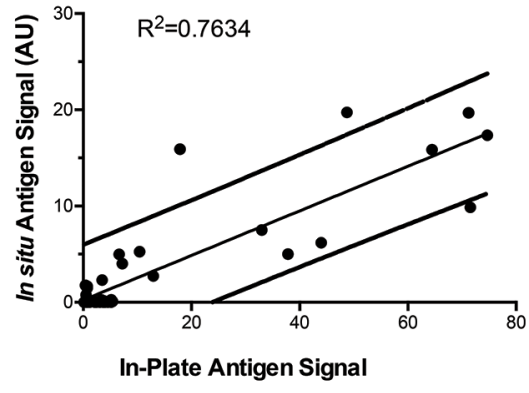

Figure 1. Antigen flow through the lymphatic chain can be monitored with infrared fluorescent dye-labeled antigen. (A) Keyhole limpet hemocyanin $(\mathrm{KLH})$ was labeled with $800 \mathrm{CW}$ and run on a polyacrylamide gel. (B) A linear correlation was found between the amount of $\mathrm{KLH}-800$ and the fluorescent signal intensity (Fluorescent antigen signal is expressed as "arbitrary units (A.U.)). (C, D) Increasing amounts of $\mathrm{KLH}-800$ were injected subcutaneously into the dorsum of the hind paw of a Balb/c mouse. The animal was imaged after $1 \mathrm{~h}$; increasing amounts of KLH-800 correlated with an increasing fluorescent signal located at both the (C) popliteal and (D) sciatic lymph nodes. Data represent mean $\pm S E M, n=5$ per concentration. A similar increase in signal was observed in (E) popliteal and (F) sciatic lymph nodes extracted from mice injected with increasing amounts of KLH-800 and minced into T-Per. Data represent mean \pm SEM, $\mathrm{n}=5$ per concentration. (G) A linear correlation was found between in situ signal and in-plate signal with an R-squared value of 0.7634 . The dotted line indicates the $95 \%$ prediction band. 
be used to monitor the localization and concentration of immunized antigen in the lymphatic system.

\section{Antigen Flow Through Peripheral Lymph Nodes Is Restricted in Immunized Animals}

To investigate antigen trafficking in previously immunized mice, animals received two intraperitoneal injections of unlabeled antigen (KLH) given $2 \mathrm{wks}$ apart, with alum as an adjuvant. Two weeks after the second injection, KLH$800 \mathrm{CW}$ was injected into the dorsum of the hind paw. Prior immunization induced restriction of the antigen flow through the lymphatic system. As compared with naïve mice, a significant decrease in the antigen signal was observed in the sciatic lymph node in immunized mice (naïve, $54.34 \pm 8.840, \mathrm{n}=5$ versus KLH-immunized, $9.730 \pm 3.715, \mathrm{n}=5$, $p<.001$ by $t$ test) (Figures 2A, B). Notably, we observed reduced signals in the popliteal lymph node, indicating that antigen flow was restricted through the lymphatic system, first from the dorsal foot to the popliteal lymph node and then to the sciatic lymph node.

To assess the specificity of this restriction, mice were immunized with either KLH or OVA and then challenged with labeled KLH (KLH-800CW) or OVA (OVA-680LT). Immunization with KLH restricted the subsequent challenge with labeled KLH (Figure 2C, second panel, KLH in green) but failed to restrict an unrelated antigen, labeled OVA (Figure 2C, fourth panel, OVA in red). However, immunization with OVA led to subsequent restriction of OVA (Figure 2C, third panel, OVA in red). Together, these results indicate that an antigen-specific "memory" leads to subsequent restriction of the same antigen.

To determine the durability of the "memory," mice were challenged with labeled KLH at various time points

\section{A}

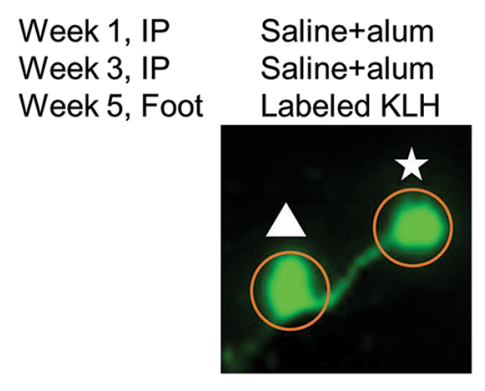

B
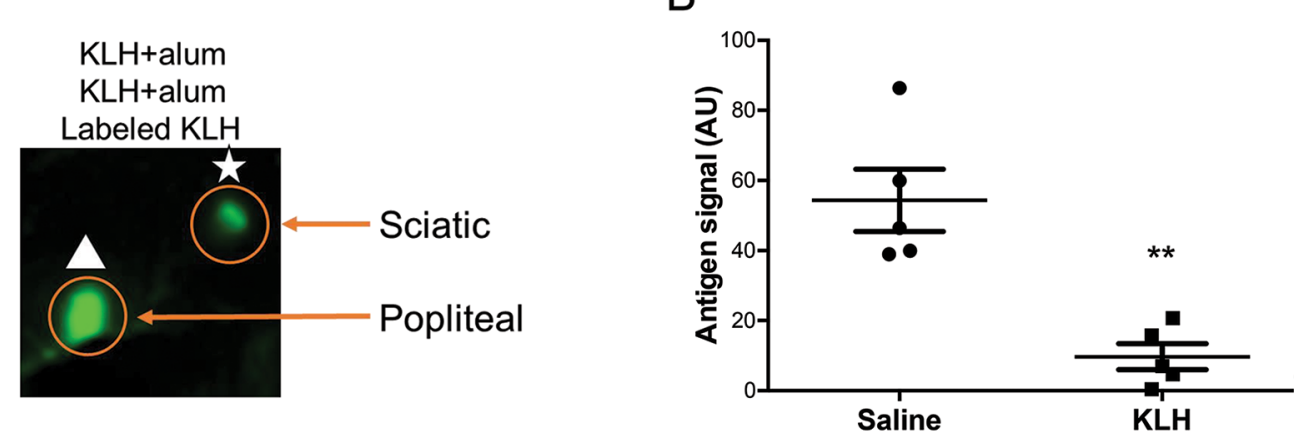

$\mathrm{D}$

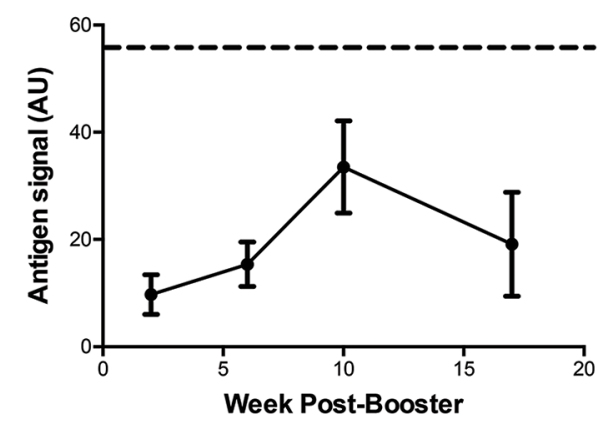

Figure 2. Antigen flow is restricted in immunized mice upon re-exposure to that antigen. $(A, B)$ Mice were immunized twice with intraperitoneal injections of KLH and alum or saline and alum. Two weeks after the second injection, $200 \mu \mathrm{g}$ of KLH-800CW was injected subcutaneously into the dorsum of the hind paw. (A) A lesser antigen signal was observed in the sciatic lymph nodes of KLH-immunized mice as compared with naïve mice. Images are representative; triangles indicate popliteal lymph nodes and stars represent sciatic lymph nodes. (B) Data shown are individual values (naïve, $54.34 \pm 8.840, n=5$ versus KLH-immunized, $9.730 \pm 3.715, n=5, p<.001$ by $t$ test). (C) This effect was specific to the immunized antigen, as OVA signal injected in a mouse immunized to KLH was not reduced, but OVA injected into OVA-immunized mice was reduced. Images are representative of five animals per group. (D) Antigen signal in the sciatic lymph node remained less in mice injected in the foot for at least 17 wks after booster injection. Antigen signal from the sciatic lymph node in animals immunized with $\mathrm{KLH}-800 \mathrm{CW}$ was determined at various time points following booster injection with $\mathrm{KLH}$. The hashed line at the top of the figure represents the mean antigen signal detected in sciatic lymph nodes of saline-immunized control animals, as displayed in Figure 2B. Data represent mean \pm SEM. Dotted line indicates average naïve mouse antigen signal. $n=5$ to 8 . 
up to 17 wks following the second immunization. Control animals received immunization with saline. A consistent decrease in antigen concentration was observed in the sciatic lymph node in immunized mice as compared with the naïve (saline-immunized) controls. This indicates that immunization with an antigen confers a long-lasting restriction of that antigen upon subsequent challenge (Figure 2D).

\section{Antigen Flow Restriction Is Dependent on Sensory Neural Input}

To determine whether an active neuronal input is required for restriction of antigen, neuronal activation was blocked locally prior to challenge with labeled antigen using bupivacaine, a sodium channel blocker that inhibits afferent neural signals. Animals were immunized with intraperitoneal injections of KLH with $50 \%$ alum twice, 2 wks apart. Two weeks after the second injection, bupivacaine was injected at the sciatic and femoral nerves, the main nerve bundles innervating the leg, prior to challenge with labeled KLH. In control animals, the same volume of saline was injected at each location. Blocking of the nerves in immunized mice was sufficient to increase antigen signal in the sciatic lymph node significantly (Figures 3A, B) (saline, $31.45 \pm 3.759, \mathrm{n}=10$; bupivacaine, 45.65 $\pm 5.350, \mathrm{n}=9, p<.05$ by $t$ test).

Direct binding of bacterial products to nociceptors induced hyperalgesia and modulated the local immune response, and ablation of $\mathrm{Na}_{\mathrm{V}} 1$.8-lineage neurons increased local immune infiltration and lymphadenopathy of the draining lymph node (9). To determine the role of nociceptors in modulating the transit of labeled antigen, we generated $\mathrm{Na}_{\mathrm{V}} 1.8-\mathrm{Cre}$ / diphtheria toxin A (DTA) mice to specifically ablate these cells. $\mathrm{Na}_{\mathrm{V}} 1.8-\mathrm{Cre}^{ \pm} \mathrm{DTA}^{ \pm}$ mice are devoid of all $\mathrm{Na}_{\mathrm{V}}$ 1.8-expressing nociceptors (15). Restriction of antigen flow to the sciatic lymph node after immunization was abolished in $\mathrm{Na}_{\mathrm{V}} 1.8-\mathrm{Cre}^{ \pm} \mathrm{DTA}^{ \pm}$mice, indicating that $\mathrm{Na}_{\mathrm{V}} 1.8$-lineage nociceptor neurons play a key role in modulating antigen flow through lymphatics (Figures 3C, D) (control, $15.41 \pm 3.526, \mathrm{n}=13$ versus $\mathrm{Na}_{\mathrm{V}} 1.8$ Cre $^{ \pm}$DTA $^{ \pm}, 35.56 \pm 6.035, \mathrm{n}=16, p<.05$ by $t$ test). No difference was observed in naïve $\mathrm{Na}_{\mathrm{V}} 1.8-\mathrm{Cre}^{ \pm} \mathrm{DTA}^{ \pm}$mice compared with controls (data not shown). Additionally, serum IgG levels against KLH were similar in $\mathrm{Na}_{\mathrm{V}} 1.8-\mathrm{Cre}^{ \pm} \mathrm{DTA}^{ \pm}$and control mice, suggesting that these animals were not merely immunocompromised (Figures 3E-F). These data suggest that neuronal input is required for the observed restriction of flow of immunized antigens.

Next we used targeted diphtheria toxin-based cell ablation to investigate whether transient receptor potential channel vanilloid 1 (TRPV1)-expressing neurons play a role in modulating antigen flow. TRPV1 is expressed by a subset of $\mathrm{Na}_{\mathrm{V}}$ 1.8-expressing nociceptive neurons and is essential for transmitting nociceptive thermal stimuli and establishing and maintaining inflammatory hyperalgesia (16). TRPV1-Cre ${ }^{ \pm} \mathrm{DTA}^{ \pm}$ mice and control littermates developed restriction of antigen trafficking to the sciatic lymph node. However, immunized $\mathrm{Na}_{\mathrm{V}} 1.8-\mathrm{Cre}^{ \pm} \mathrm{DTA}^{ \pm}$mice exhibited increased antigen concentration in the sciatic lymph node (Figure 3G) (control, $6.783 \pm 1.290, \mathrm{n}=9$; TRPV1-DTA, $6.345 \pm$ 1.547, $\mathrm{n}=10 ; \mathrm{Na}_{\mathrm{V}} 1.8-\mathrm{DTA}, 43.93 \pm 15.57$, $\mathrm{n}=6, p<.001$ by one-way ANOVA Bonferroni post-test). These data indicate that a subset of $\mathrm{Na}_{\mathrm{V}} 1.8$ neurons that lack TRPV1 expression plays an important role in modulating antigen restriction.

\section{Neuronal Stimulation Restricts Antigen Flow}

To functionally explore the effect of direct stimulation of the nerve fibers innervating the lymph nodes in mediating antigen restriction, we stimulated the femoral and sciatic nerves in the popliteal fossa using electrical or magnetic stimulation. Electrical stimulation $(-5 \mathrm{~V}$ constant, 0.75 msec pulse duration, 20 $\mathrm{Hz}$ ) was applied adjacent to the popliteal lymph node using a monopolar needle electrode. Control animals did not receive any electrical stimulation. Animals received labeled KLH in the dorsum of the hind paw, and antigen flow through the popliteal and sciatic lymph nodes was monitored. Antigen flow to the sciatic lymph node was significantly restricted in naïve animals subjected to electrical stimulation. In contrast, animals with sham stimulation failed to restrict the antigen (sham, $114.1 \pm 16.17$, $\mathrm{n}=5$ versus electrically stimulated, 56.76 $\pm 14.38, \mathrm{n}=5, p<.05$ by $t$ test) (Figures $4 \mathrm{~A}, \mathrm{~B})$.

We next used a time-varying magnetic field with an electromagnetic coil directed to the hind leg to stimulate the local neural network in naïve animals. Sham animals received no stimulation. Following stimulation, animals were subjected to challenge with KLH-800CW in the hind paw. Similar to the electrical stimulation, activation of the neural network using magnetic stimulation significantly restricted antigen flow to the sciatic lymph node in naïve animals as compared with sham stimulated animals (sham stimulated, $55.99 \pm 4.993, \mathrm{n}=10$ versus magnetically stimulated, $30.87 \pm 4.169, \mathrm{n}=12$, $p<.001$ by $t$ test) (Figures 4C, D). These data clearly demonstrate that direct neuronal activation regulates antigen flow in the lymph nodes.

\section{Neuronal Fc Receptors Play a Role in Antigen Restriction}

The Fc receptors (FcRs) play an important role in adaptive immune responses by binding antigen-antibody complexes. In addition to immune effector cells, FcRs are also expressed on neurons $(17,18)$. We therefore reasoned that antigen-antibody interactions with FcR might underlie the restriction mechanism. To determine the role of antigen-specific antibodies and antigen complexes in mediating antigen restriction, we utilized a passive immunization model. Animals received intraperitoneal administration of polyclonal anti-OVA antibodies and were challenged $24 \mathrm{~h}$ later in the dorsum of the hind paw with labeled OVA-800CW or KLH-800CW. Administration of OVA-specific antibodies significantly decreased transit of 
A

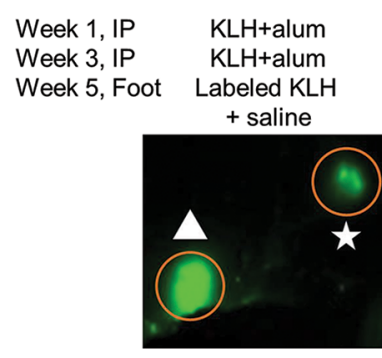

C

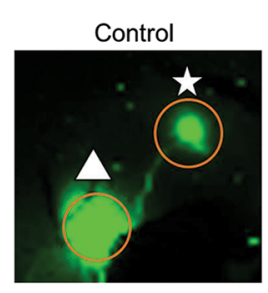

E

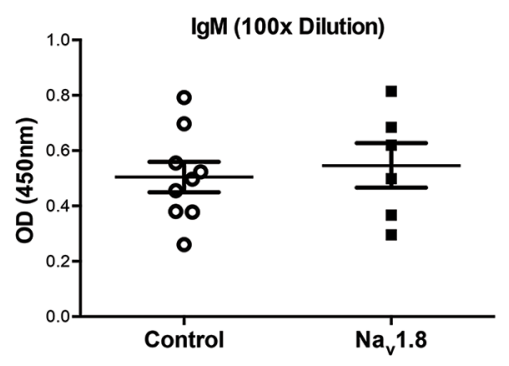

B
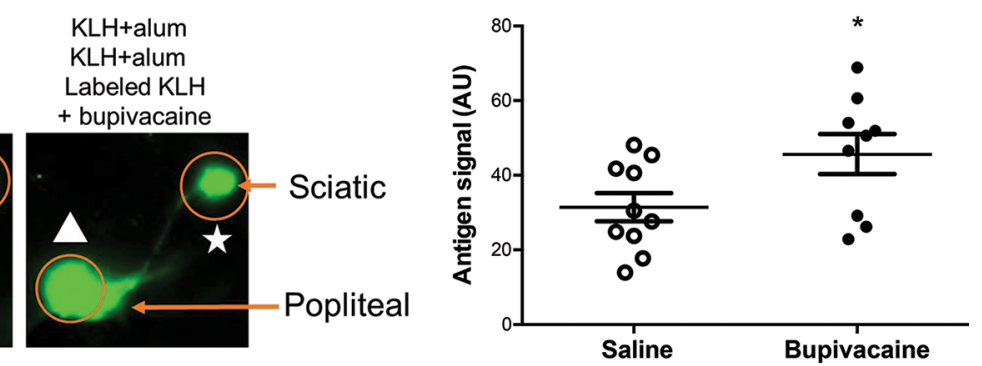

D

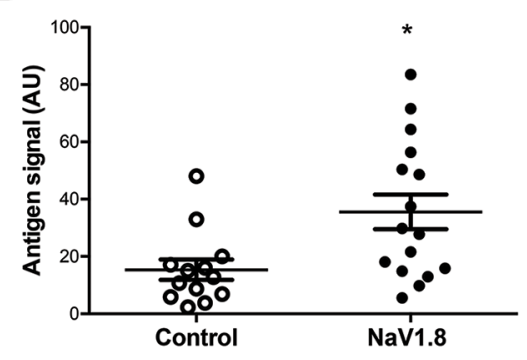

$\mathrm{F}$

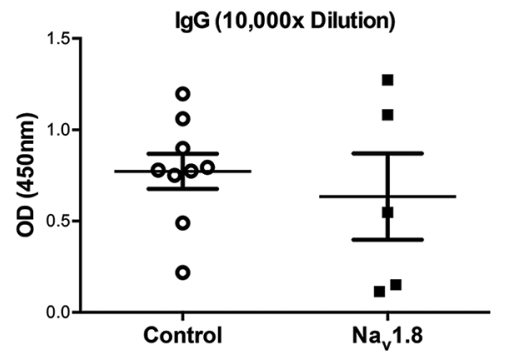

G

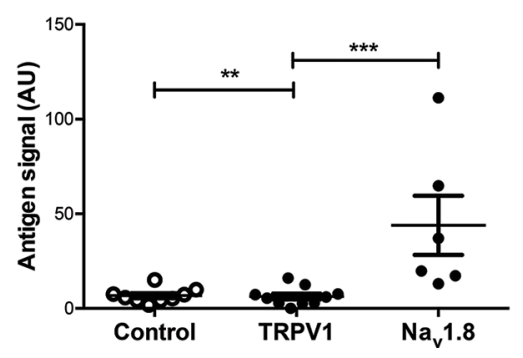

Figure 3. Antigen flow restriction is dependent on sensory neural input. (A, B) Mice immunized with KLH were injected with bupivacaine at the femoral and sciatic nerves. Upon challenge with $\mathrm{KLH}-800 \mathrm{CW}$, an increase in antigen signal was seen in the sciatic lymph node. (A) Images are representative; triangles indicate popliteal lymph nodes and stars represent sciatic lymph nodes. (B) Antigen signal in the sciatic lymph node in individual animals is shown here. (mean \pm SEM: saline, $31.45 \pm 3.759, n=10$, bupivacaine, $45.65 \pm 5.350, n=9$, $p<.05$ by t test). (C, D) Nav1.8-DTA mice were immunized with $\mathrm{KLH}$. Images are representative; triangles indicate popliteal lymph nodes and stars represent sciatic lymph nodes. (D) Antigen signal in the sciatic lymph node in individual animals is shown here. (mean \pm SEM control, $15.41 \pm 3.526, n=13$; Na $1.8-D T A, 35.56 \pm 6.035, n=16, p<.05$ by $t$ test). (E, F) No difference in the circulating levels of (E) lgM and (F) IgG was seen in Na 1.8-DTA mice (lgM, $\mathrm{Na}_{v}$ 1.8-depleted, $0.5044 \pm 0.05477, \mathrm{n}=9$ versus control, $0.5466 \pm 0.08038, \mathrm{n}=6$ ) (lgG, $\mathrm{Na}_{v}$ 1.8-depleted, $0.7737 \pm 0.09626, \mathrm{n}=9$ versus control, $0.6340 \pm 0.2365, \mathrm{n}=5$ ). (G) Immunized TRPV 1-DTA, Nav1.8-DTA and littermate control mice were injected with $\mathrm{KLH}-800 \mathrm{CW}$ in the hind paw. The increase in antigen seen in Na $1.8-D T A$ mice was not recapitulated in TRPV1-DTA mice in the sciatic lymph node (control, $6.783 \pm 1.290, n=9$; TRPV1-DTA, $6.345 \pm 1.547, n=10 ; N a_{V} 1.8-D T A, 43.93 \pm 15.57, n=6$, $p<.001$ by one-way ANOVA Bonferroni post-test). Data represent individual values and mean \pm SEM. 
A

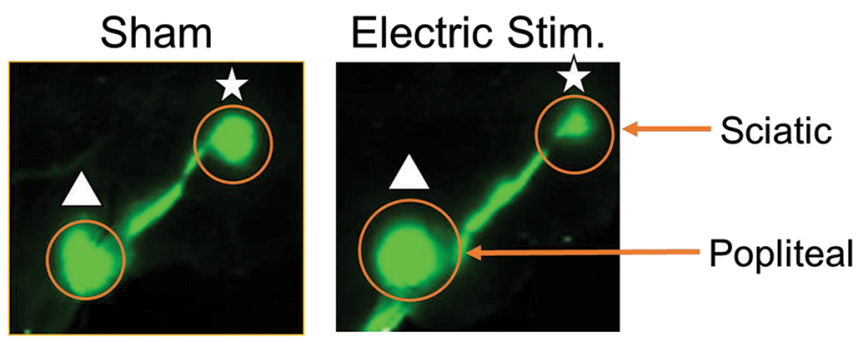

C

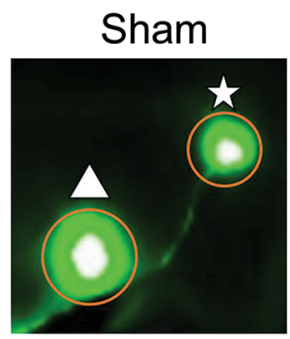

B

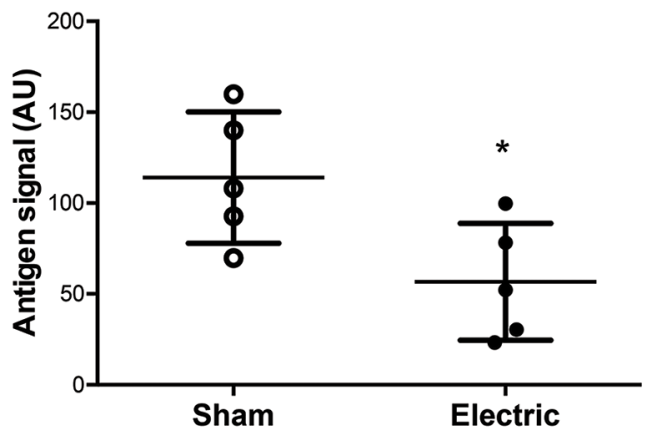

D

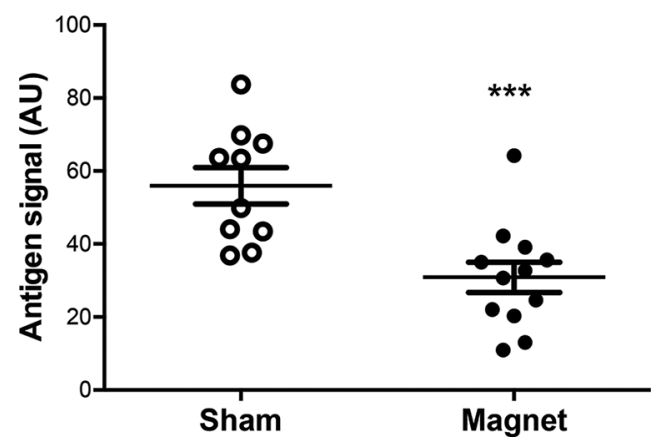

Figure 4. Stimulation of neuronal activity restricts antigen flow. (A, B) Electric stimulation of the nerves to the popliteal and sciatic lymph node restricts $\mathrm{KLH}-800 \mathrm{CW}$ flow to the sciatic lymph nodes (sham, 114.1 $\pm 16.17, \mathrm{n}=5$ versus electrically stimulated, $56.76 \pm 14.38, \mathrm{n}=5$, $p<.05$ by + test). (A) Images are representative. (B) Antigen signal in the sciatic lymph node in individual animals is shown here. (C, D) A noninvasive magnetic field was applied to the hind legs of nailve mice to induce nerve activity, followed by injection of KLH-800CW. In magnetically stimulated animals, lower levels of antigen were observed in the sciatic lymph nodes (sham, 55.99 $\pm 4.993, \mathrm{n}=10$ versus magnetically stimulated, $30.87 \pm 4.169, n=12, p<.001$ by $t$ test). (C) Images are representative; triangles indicate popliteal lymph nodes and stars represent sciatic lymph nodes. (D) Antigen signal in the sciatic lymph node in individual animals is shown here.

OVA antigen to the sciatic lymph nodes. In contrast, anti-OVA antibodies failed to restrict KLH transport, indicating antigen specificity of the antibody-induced lymph node transit $(\mathrm{KLH}, 32.95 \pm 7.741$, $\mathrm{n}=10$ versus OVA, $6.483 \pm 1.461, \mathrm{n}=9$ ) (Figure 5A).

To explore the consequences of deleting $\mathrm{FcR}$ expression, we analyzed antigen restriction in naïve and immunized $\mathrm{FcR}$ $\mathrm{KO}$ mice. Comparable levels of KLH signals were observed in naïve Balb/c and FCR KO mice, indicating that antigen trafficking is not different in naïve mice lacking FcR (Balb/c, $62.50 \pm 5.614, \mathrm{n}=$ 8 versus FCR KO, $71.48 \pm 8.197, \mathrm{n}=6$ ) (Figure 5B). In contrast, a significantly increased level of antigen was observed in the sciatic lymph nodes of KLHimmunized FcR KO compared with wild-type mice (Balb/c, $15.76 \pm 2.721$, $\mathrm{n}=10$ versus FCR KO, $44.29 \pm 6.500$, $\mathrm{n}=11, p<.001$ by $t$ test) (Figure 5C). Moreover, in mice immunized with $\mathrm{KLH}$, antigen and $\mathrm{FC}_{\mathrm{C}}$ receptors colocalized on PGP9.5 + ve neuronal tissue at the site of injection. (Figure 5D). Together, these data suggest that neuronal Fc receptors participate in the neural restriction of immunized antigen.

\section{DISCUSSION}

Here we have identified a novel neural reflex circuit that mediates restriction of antigen transit in immunized animals (Figure 6). We demonstrate that challenge with an immunized antigen initiates a neural response in TRPV1 $\mathrm{Na}_{\mathrm{V}} 1.8^{+}$neurons, leading to an effector response, which results in restriction of antigen transit from local lymph node A to lymph node B (e.g., popliteal to sciatic lymph node). Direct activation of the local neural network recapitulates the antigen restriction in a naïve mouse in the absence of prior immunization. In contrast, selective neural blockade abrogates the antigen-specific restriction in immunized animals.

We and others have identified reflex neural circuits that modulate the output of the immune system in other organ systems. In Caenorhabditis elegans, neurons can regulate innate immunity, 
A

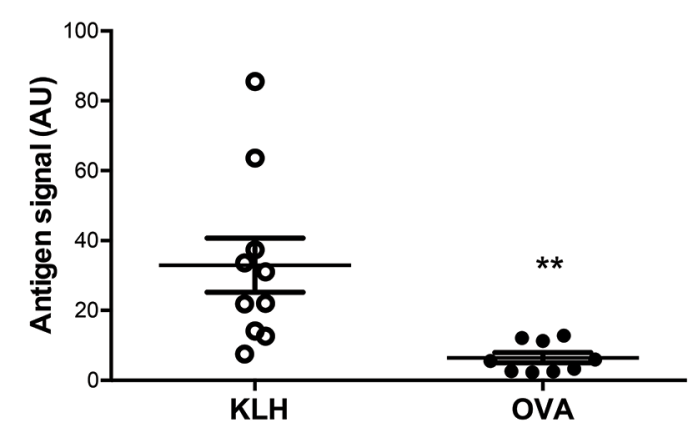

C

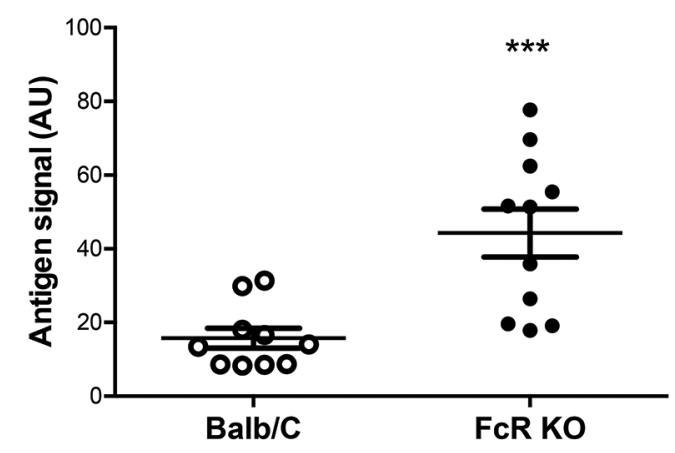

B
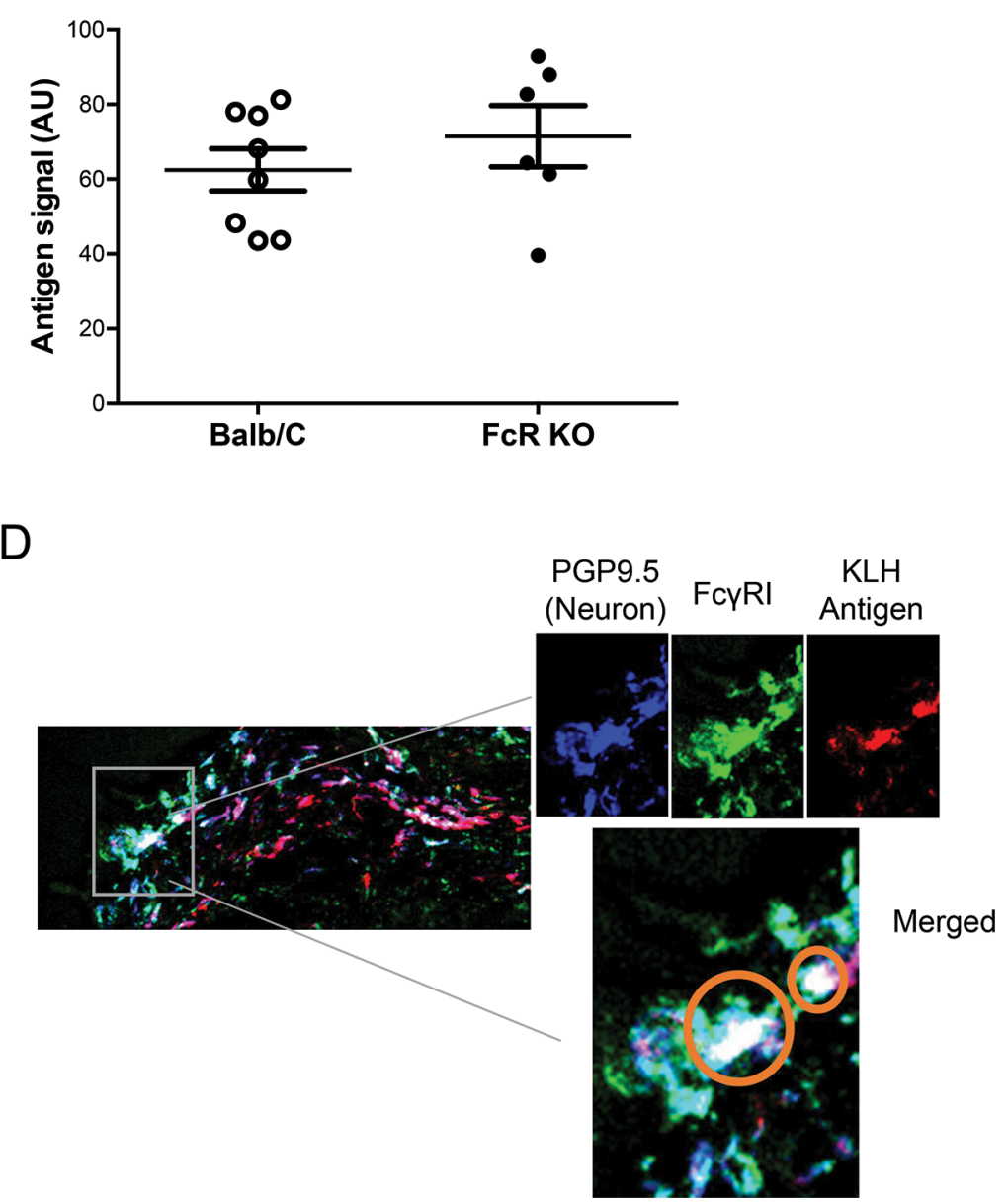

Figure 5. Neuronal Fc receptors play a role in antigen restriction. (A) Naïve mice were injected intraperitoneally with rabbit polyclonal anti-OVA antibodies, and $24 \mathrm{~h}$ later OVA-800CW or KLH-800CW was injected in the dorsum of the hind paw. The amount of OVA antigen was significantly decreased compared with $\mathrm{KLH}$ in the sciatic lymph nodes (mean $\pm \mathrm{SEM}: \mathrm{KLH}, 32.95 \pm 7.741, \mathrm{n}=10$ versus OVA, $6.483 \pm 1.461, n=9$ ). (B) Antigen trafficking is not different in naïve FCR KO mice. Naïve Balb/c and FcR KO mice were injected subcutaneously in the hind paw with $\mathrm{KLH}-800 \mathrm{CW}$. No difference was seen in the antigen signals of nailve Balb/c and FcR KO mice in the sciatic Iymph nodes (mean \pm SEM: Balb/c, $62.50 \pm 5.614, n=8$ versus FCR KO, $71.48 \pm 8.197, n=6$ ). (C) Increased antigen levels were observed in immunized FCR KO mice challenged with KLH-800CW (mean \pm SEM Balb/c, $15.76 \pm 2.721, \mathrm{n}=10$ versus FCR $\mathrm{KO}, 44.29 \pm 6.500, \mathrm{n}=11$, $p<.001$ by t test). (D) Colocalization of antigen and Fc receptors on PGP9.5 + ve neuronal tissue at the site of injection. Mice immunized with KLH were challenged with KLH-A647 in the dorsum of the hind paw. After $1 \mathrm{~h}$, skin around the injection site was excised, frozen in optimal cutting temperature media and sectioned at $10 \mu \mathrm{m}$. Tissue slices were mounted on slides, then stained with antibodies against PGP9.5 and FcyRl. Images were obtained on a laser-scanning confocal microscope. Images shown are representative of slices from three different animals. Blue indicates PGP9.5, green indicates Fc $\gamma \mathrm{Rl}$, red indicates signal from KLH-A647 and white in the merged image indicates colocalization of all three signals. Orange circles indicate regions of interest.

indicating the early evolutionary origins of the regulation of innate immune responses by the nervous system $(19,20)$. In mammals, neural networks regulate immune responses in the spleen, gut, lungs, central nervous system and even cancer tissues (21-25). It has been proposed that neural activation in the lymphoid organs maintains the integrity of the antibody response (21), but a direct modulation of antigen transit, as revealed here, has not been described. Identification of a neural reflex mechanism that contributes to antigen transit reveals a previously unknown mechanism for antigen restriction. The observation that a depletion of $\mathrm{Na}_{\mathrm{V}} 1.8^{+}$neurons results in an abrogation of antigen restriction indicates that there is a role for nociceptor neurons in antigen recognition and restriction. Nociceptors have previously been linked to mediating bacteria-induced pain, modulating allergic inflammatory responses and potentiating adaptive 


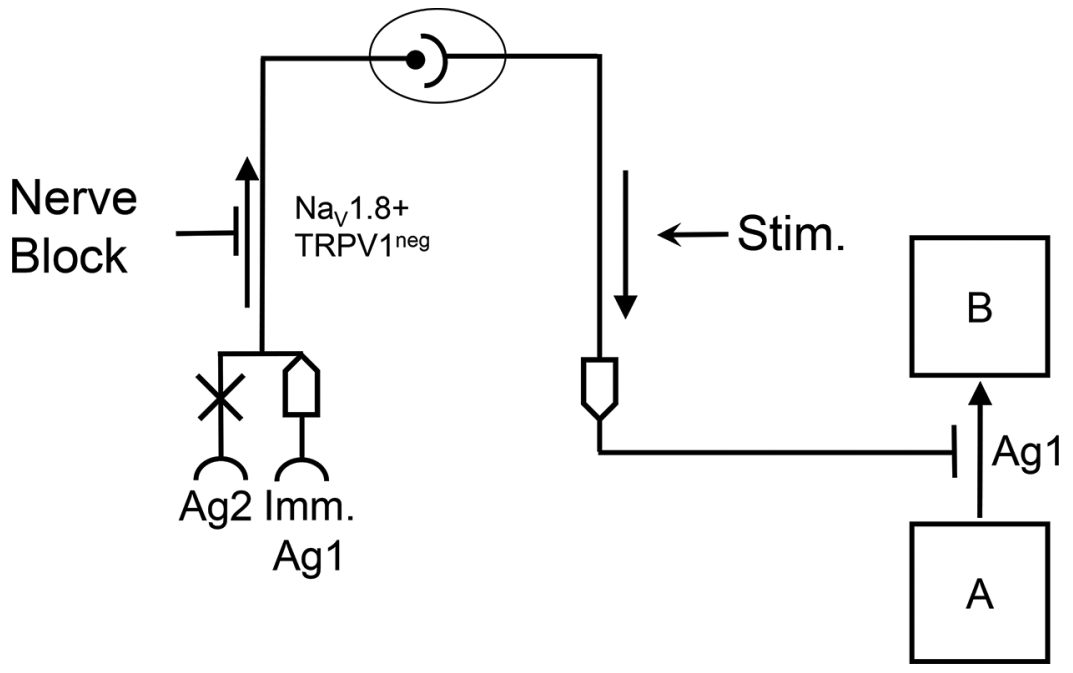

Figure 6. A model diagram depicting the antigen-restriction reflex. A neuronal pathway distinguishes between novel and immunized antigens, sending a nerve signal through $\mathrm{Na}_{V} 1.8$ + ve, TRPV $1^{\text {neg }}$ neurons, which is transmuted into a motor signal leading to restriction of antigen flow from lymph node A to lymph node B (e.g., popliteal to sciatic lymph node).

immune responses $(9,10,12)$. Here we show that nociceptors are crucial for antigen-specific restriction of antigen transport through the lymphatic system. Furthermore, our data provide direct evidence that expression of FcR is essential for mediating antigen-specific restriction through lymphatics. It has been argued that a subpopulation of nociceptive neurons expresses functional $\mathrm{FcR}_{\mathrm{C}}$, and that activation of this receptor by antigenantibody complexes increases neuronal excitability (26). The current study suggests that antigen-specific activation of nociceptors via FcR contributes to antigen restriction and transport.

Our data reveal a potential mechanism to control the spread of pathogenic bacteria or micrometastases. Upon infection, bacteria routinely disseminate through the lymphatic system, eventually leading to a systemic infection. It is plausible to selectively activate the local neural network of the neurons innervating the draining lymph node to prevent bacteremia and sepsis. Upon activation, neurons convey information through temporal patterns of action potentials and graded membrane potential shifts (27-29). It is conceivable to record and decode the sensory signals activated during the antigen restriction reflex, which could have implications for developing novel therapies for bacterial infection. Indeed, distinct signals were recorded in the sciatic nerve of the immunized animals after antigen challenge in the foot compared with naïve animals (data not shown). The lymphatic system also plays a crucial role in cancer metastasis (30-32). Many therapeutic courses involve lymphatic mapping to determine which lymph nodes are draining the site of a primary tumor (33-38). These lymph nodes are then monitored for development of new micrometastases. An improved understanding of the role of the neural network in modulating lymph node trafficking suggests that limiting micrometastases by activating the local neural network could provide a novel therapy for cancer metastasis.

The lymphatic system plays a vital role in conveying free antigen and antigen-presenting cells from the tissues to local draining lymph nodes for immune surveillance and activation. Lymphatic flow through the lymph vessels is modulated by the intrinsic contractile property of the lymphatic smooth muscles. Changes in the local microenvironment regulate the function of the smooth muscles and modulate the lymphatic flow. During critical illness, local and systemic inflammation play an important role in initiating and establishing muscle weakness (39-41). Although inflammatory responses induced by an antigen challenge in immunized animals can lead to a reduction in muscle contractions, resulting in restriction of lymphatic flow and antigen trafficking, these effects require chronic exposure to inflammatory mediators (39). Here, we monitored antigen transport in an acute setting. It is plausible that delayed type hypersensitivity (DTH) could lead to increased pain and immobility in an acute setting. However, these responses usually peak within hours. In animals with KLHinduced DTH responses, the peak swelling in response to $\mathrm{KLH}$ administration in the foot is observed after $24 \mathrm{~h}$ (42). In contrast, our data demonstrate that prior immunization with an antigen restricts antigen flow within minutes in an immunized animal, indicating that DTH does not mediate these responses. Moreover, direct electrical stimulation induced antigen restriction in naïve animals, further confirming the role of neural activation in modulating lymphatic function.

\section{CONCLUSION}

The discovery and characterization of a novel neural circuit wherein sensory and motor nerves regulate antigen trafficking from distal to proximal lymph nodes provides additional insight into the interplay between the nervous and immune systems. Ongoing studies to understand the sensory signals and molecular mechanisms of this antigen restriction reflex might reveal direct sensory and effector roles of the nervous system in antigen restriction and provide insights that have implications for developing bioelectronic therapies.

\section{DISCLOSURE}

The authors declare that they have no competing interests as defined by Bioelectronic Medicine, or other interests that might be perceived to influence the results and discussion reported in this paper. 


\section{REFERENCES}

1. Panuncio AL, De La Peña S, Gualco G, Reissenweber N. (1999) Adrenergic innervation in reactive human lymph nodes. J. Anat. 194(Pt 1):143-46.

2. Wulfing C, Gunther HS. (2015) Dendritic cells and macrophages neurally hard-wired in the lymph node. Sci. Rep. 5:16866.

3. Kannan Y, Bienenstock J, Ohta M, Stanisz M, Stead RH. (1996) Nerve growth factor and cytokines mediate lymphoid tissue-induced neurite outgrowth from mouse superior cervical ganglia in vitro. J. Immunol. 157:313-20.

4. Kinkelin I, Mötzing S, Koltenzenburg M, Bröcker EB. (2000) Increase in NGF content and nerve fiber sprouting in human allergic contact eczema. Cell Tissue Res. 302:31-37.

5. Novotny GEK, Heuer T, Schöttelndreier A, Fleisgarten C. (1994) Plasticity of innervation of the medulla of axillary lymph nodes in the rat after antigenic stimulation. Anat. Rec. 238:213-24.

6. Benschop RJ, Rodriguez-Feuerhahn M, Schedlowski M. (1996) Catecholamine-Induced Leukocytosis: Early Observations, Current Research, and Future Directions. Brain Behav. Immun. 10:77-91.

7. McHale NG, Thornbury KD. (1990) Sympathetic stimulation causes increased output of lymphocytes from the popliteal node in anaesthetized sheep. Exp. Physiol. 75:847-50.

8. Moore TC. (1984) Anesthesia-associated depression in lymphocyte traffic and its modulation. Am. J. Surg. 147:807-12.

9. Chiu IM, Heesters BA, Ghasemlou N, et al. (2013) Bacteria activate sensory neurons that modulate pain and inflammation. Nature. 501:52-57.

10. Talbot S, Abdulnour R-EE, Burkett PR, et al. (2015) Silencing Nociceptor Neurons Reduces Allergic Airway Inflammation. Neuron. 87:341-54.

11. Kashem SW, Riedl MS, Yao C, Honda CN, Vulchanova L, Kaplan DH. (2015) Nociceptive Sensory Fibers Drive Interleukin-23 Production from CD301b ${ }^{+}$Dermal Dendritic Cells and Drive Protective Cutaneous Immunity. Immunity. 43:515-26

12. Riol-Blanco L, Ordovas-Montanes J, Perro M, et al. (2014) Nociceptive sensory neurons drive interleukin-23-mediated psoriasiform skin inflammation. Nature. 510:157-61.

13. Agarwal N, Offermanns S, Kuner R. (2004) Conditional gene deletion in primary nociceptive neurons of trigeminal ganglia and dorsal root ganglia. Genesis. 38:122-29.

14. Committee for the Update of the Guide for the Care and Use of Laboratory Animals, Institute for Laboratory Animal Research, Division of Earth and Life Studies, National Research Council of the National Academies. (2011) Guide for the Care and Use of Laboratory Animals. 8th ed. Washington, DC: National Academies Press.

15. Abrahamsen B, Zhao J, Asante CO, et al. (2008) The Cell and Molecular Basis of Mechanical, Cold, and Inflammatory Pain. Science. 321:702-05.
16. Chiu IM, von Hehn C, Woolf CJ. (2012) Neurogenic inflammation and the peripheral nervous system in host defense and immunopathology. Nat. Neurosci. 15:1063-67.

17. Fuller JP, Stavenhagen JB, Teeling JL. (2014) New roles for $\mathrm{Fc}_{\mathrm{C}}$ receptors in neurodegeneration: the impact on immunotherapy for Alzheimer's disease. Front. Neurosci. 8:235.

18. van der Kleij H, Charles N, Karimi K, et al. 2010. Evidence for neuronal expression of functional Fc (epsilon and gamma) receptors. J. Allergy Clin. Immunol. 125:757-60.

19. Aballay A. 2009. Neural regulation of immunity: role of NPR-1 in pathogen avoidance and regulation of innate immunity. Cell Cycle. 8:966-69.

20. Sun J, Singh V, Kajino-Sakamoto R, Aballay A. 2011. Neuronal GPCR Controls Innate Immunity by Regulating Noncanonical Unfolded Protein Response Genes. Science. 332:729-32.

21. Mina-Osorio P, et al. (2012) Neural signaling in the spleen controls B-cell responses to bloodborne antigen. Mol. Med. 18(4):618-27.

22. Rosas-Ballina M, Ochani M, Parrish WR, et al. (2008) Splenic nerve is required for cholinergic antiinflammatory pathway control of TNF in endotoxemia. Proc. Natl. Acad. Sci. U.S.A. 105:11008-13

23. Matteoli G, et al. (2014) A distinct vagal anti-inflammatory pathway modulates intestinal muscularis resident macrophages independent of the spleen. Gut. 63(6):938-48.

24. Gabanyi I, Muller PA, Feighery L, Oliveira TY, Costa-Pinto FA, Mucida D. (2016) Neuroimmune Interactions Drive Tissue Programming in Intestinal Macrophages. Cell. 164:378-91.

25. Magnon C, Hall S, Lin J, Xue X, Gerber L, Freedland S, Frenette P. (2013) Autonomic nerve development contributes to prostate cancer progression. Science. 341:1-10.

26. Qu L. (2012) Neuronal Fc gamma receptor I as a novel mediator for IgG immune complexinduced peripheral sensitization. Neural Regen. Res. 7:2075-79.

27. Bialek W, Rieke F, de Ruyter van Steveninck RR, Warland D. (1991) Reading a neural code. Science. 252:1854-57.

28. Borst A, Theunissen FE. (1999) Information theory and neural coding. Nat. Neurosci. 2:947-57.

29. Stanley GB. (2013) Reading and writing the neural code. Nat. Neurosci. 16:259-63.

30. Lens MB, Dawes M, Newton-Bishop JA, Goodacre T. (2002) Management of regional lymph nodes in patients with malignant melanoma: questionnaire survey of UK current practice. Br. J. Plast. Surg. 55:372-75.

31. Fidler IJ. (2003) The pathogenesis of cancer metastasis: the "seed and soil" hypothesis revisited. Nat. Rev. Cancer. 3:453-58.

32. Shayan R, Achen MG, Stacker SA. (2006) Lymphatic vessels in cancer metastasis: Bridging the gaps. Carcinogenesis. 27:1729-38.

33. Haigh PI, Giuliano AE. (2000) Intraoperative lymphatic mapping and sentinel lymphadenec- tomy for breast cancer. Oper. Tech. Gen. Surg. 2:161-65.

34. Morton DL, Wen DR, Wong JH, et al. (1992) Technical details of intraoperative lymphatic mapping for early stage melanoma. Arch. Surg. 127:392-99.

35. Bilchik AJ, Giuliano A, Essner R, et al. (1998) Universal application of intraoperative lymphatic mapping and sentinel lymphadenectomy in solid neoplasms. Cancer J. Sci. Am. 4:351-58.

36. Ferris RL, Xi L, Raja S, et al. (2005) Molecular staging of cervical lymph nodes in squamous cell carcinoma of the head and neck. Cancer Res. 65:2147-56.

37. Park C, Seid P, Morita E, et al. (2005) Internal mammary sentinel lymph node mapping for invasive breast cancer: implications for staging and treatment. Breast J. 11:29-33.

38. Heuts EM, van der Ent FWC, von Meyenfeldt MF, Voogd C. (2009) Internal mammary lymph drainage and sentinel node biopsy in breast cancer: A study on 1008 patients. Eur. J. Surg. Oncol. 35:252-57.

39. Witteveen E, Wieske L, Verhamme C, Schultz MJ, van Schaik IN, Horn J. (2014) Muscle and nerve inflammation in intensive care unit-acquired weakness: a systematic translational review. J. Neurol. Sci. 345:15-25.

40. Zochodne DW, Bolton CF, Wells GA, et al. (1987) Critical illness polyneuropathy. A complication of sepsis and multiple organ failure. Brain. 819-41.

41. Bolton CF, Young GB, Zochodne DW. (1993) The neurological complications of sepsis. Ann. Neurol. 33:94-100.

42. Cher DJ, Mosmann TR. (1987) Two types of murine helper T cell clone. II. Delayed-type hypersensitivity is mediated by TH1 clones. J. Immunol. 138:3688-94.

Cite this article as: Hanes WM, et al. (2016) Neuronal circuits modulate antigen flow through lymph nodes. Bioelectron. Med. 3:18-28. 